\title{
Recent improvement of the LHD Thomson scattering system
}

\author{
I. Yamada ${ }^{1}$ \\ National Institute for Fusion Science \\ Oroshi 322-6, Toki, Gifu 509-5292, Japan \\ E-mail: yamadai@LHD.nifs.ac.jp
}

\section{H. Funaba, R. Yasuhara, K. Narihara, and H. Hayashi}

National Institute for Fusion Science

Oroshi 322-6, Toki, Gifu 509-5292, Japan

\begin{abstract}
The Large Helical Device (LHD) Thomson scattering system has routinely measured electron temperature $(T)$ and density $\left(n_{e}\right)$ profiles of LHD plasmas along the LHD major radius since the second LHD experiment campaign, 1998. It is one of the most powerful Thomson scattering systems in the world. During the past 15 years, we have improved the LHD Thomson scattering system for increasing data reliability and accuracy, expanding measurable temperature and density ranges, and searching new physical findings. The number of spatial points, spatial resolution and sampling rate are 144, 20 mm, and 10-100 Hz (100-10 msec), which depends on the laser repetition frequency, respectively. In the first design, the optimized $T_{e}$ and $n_{e}$ ranges of the system were set to be $T_{e}=50 \mathrm{eV}-10 \mathrm{keV}$ and $n_{e}=10^{18}-10^{21} \mathrm{~m}^{-3}$ respectively. Current measurable $T_{e}$ and $n_{e}$ ranges have been expanded as $T_{e}=1 \mathrm{eV}-30 \mathrm{keV}$ and $n_{e}=10^{18}-10^{22} \mathrm{~m}^{-3}$. To obtain accurate and reliable $T_{e}$ and $n_{e}$ data from the LHD Thomson scattering system, we tried many recalibrations and tested a few data analysis procedures. In the paper, we describe the current status of the LHD Thomson scattering system.
\end{abstract}

First EPs Conference on Plasma Diagnostics - $1^{\text {st }}$ ECPD

14-17 April 2015,

Villa Mondragone, Frascati (Rome) Italy

${ }^{1}$ Speaker 


\section{Introduction}

The Thomson scattering system is one of the most reliable diagnostic systems for measuring local electron temperatures and densities of fusion plasmas. We have developed and installed an yttrium-aluminum-garnet (YAG) laser Thomson scattering system on the large helical device (LHD), and have measured the $T_{e}$ and $n_{e}$ profiles of LHD plasmas since the LHD second experiment campaign (1998). [1-3] The LHD Thomson scattering system has following the features: multilaser system using high-repetition YAG lasers, light collection optics based on a large spherical mirror, and multichannel polychromators. The schematic diagram of the LHD Thomson scattering system is shown in Fig.1. [3] The laser beam travels through LHD plasmas along the LHD major radius at a horizontally elongated section. Thomson scattered light is collected with a large spherical mirror, reflected onto optical fibers with a core diameter of 2 $\mathrm{mm}$, and transmitted to 144 polychromators. First we constructed 144 five-wavelength channel polychromators. The optimized $T_{e}$ range of them was set to be $50 \mathrm{eV}-10 \mathrm{keV}$. Now we have three types of polychromators; a low-temperature type (edge region), a standard type (core region), and a high-temperature type (central region). All of them have an additional Rayleigh scattering channel to determine absolute $n_{e}$. In the following sections, the current status of the LHD Thomson scattering system is described.

\section{Laser System}

In the initial phase, five $0.5 \mathrm{~J} / 50 \mathrm{~Hz}$ lasers (Continuum 8050) were installed. [4] Currently a $1.6 \mathrm{~J} / 30 \mathrm{~Hz}$ laser (Continuum 9030), two $2 \mathrm{~J} / 10 \mathrm{~Hz}$ lasers (Thales 230/10), and a $0.5 \mathrm{~J} / 50 \mathrm{~Hz}$

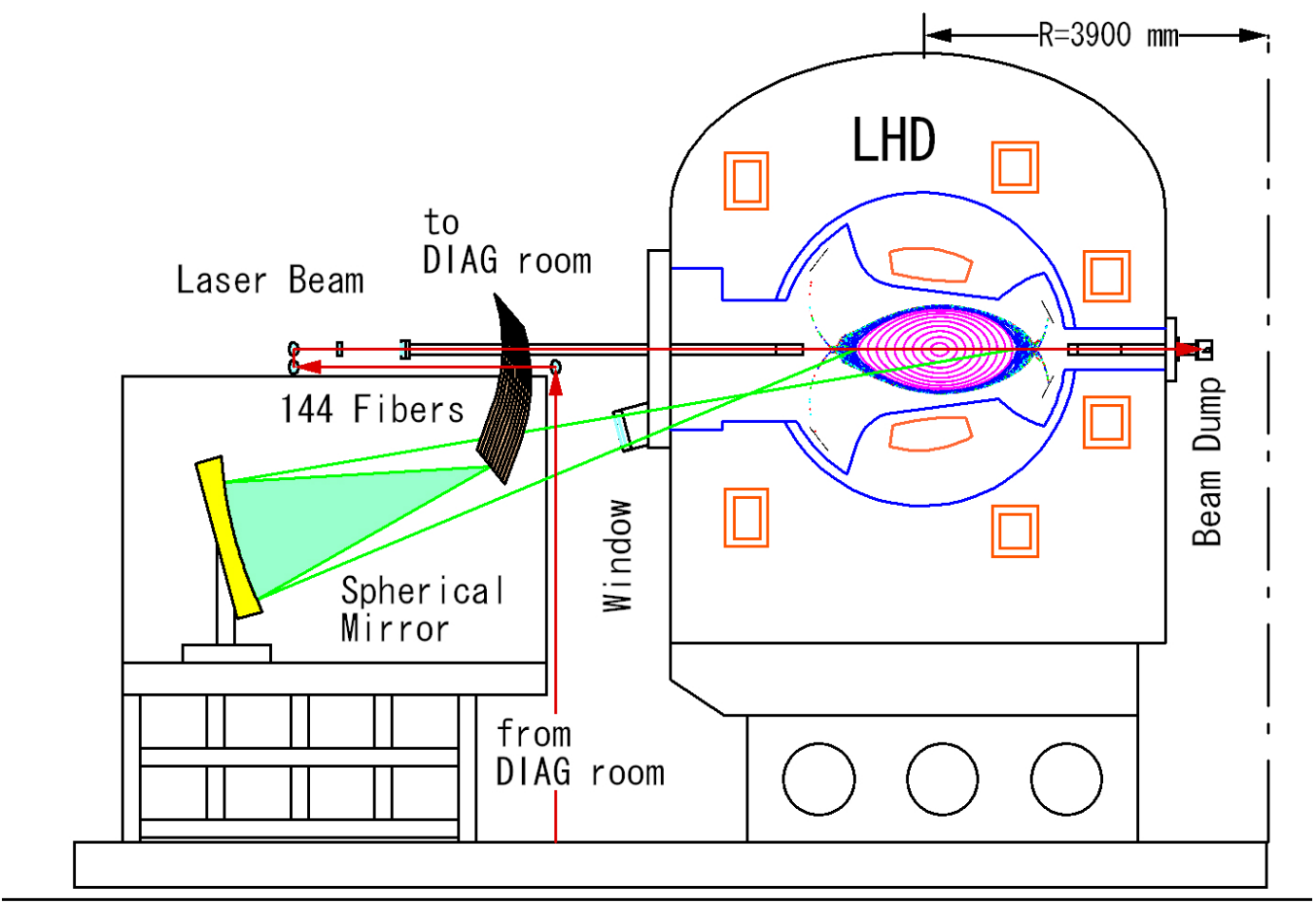

Fig.1. Schematic diagram of the LHD Thomson scattering system. 
laser (Continuum 8050) are available. In each laser system, a beam telescope optics has been installed to adjust beam diameter and divergence. In the initial phase, we developed beam packing mirrors which are similar to that developed by the DIII-D Thomson scattering team to pack several laser beams. [5] Recently, we have installed a new beam merging optics for two lasers to establish a complete coaxial beam transport. [6] The system is based on the polarization control technique. Figure 2 shows the schematics diagram. The both polarizations of laser L1 and L2 are horizontal just after the laser units. The polarizers P1 and P2 are used as polarization purifiers. The polarizations of the two beams after P1 and P2 are almost completely horizontal, better than 100:1. The polarization of the laser L1 is rotated to vertical by a $\lambda / 2$ waveplate so as to go through the polarizer P3. The beam direction and position of the beam \#1 are adjusted with the steering mirror M1. On the other hand, the laser beam $\# 2$ is reflected by the polarizer P3, and the beam direction of the beam \#2 is adjusted with the polarizer P3. Thus, the beam axes of the two laser beams can be in a coaxial configuration.

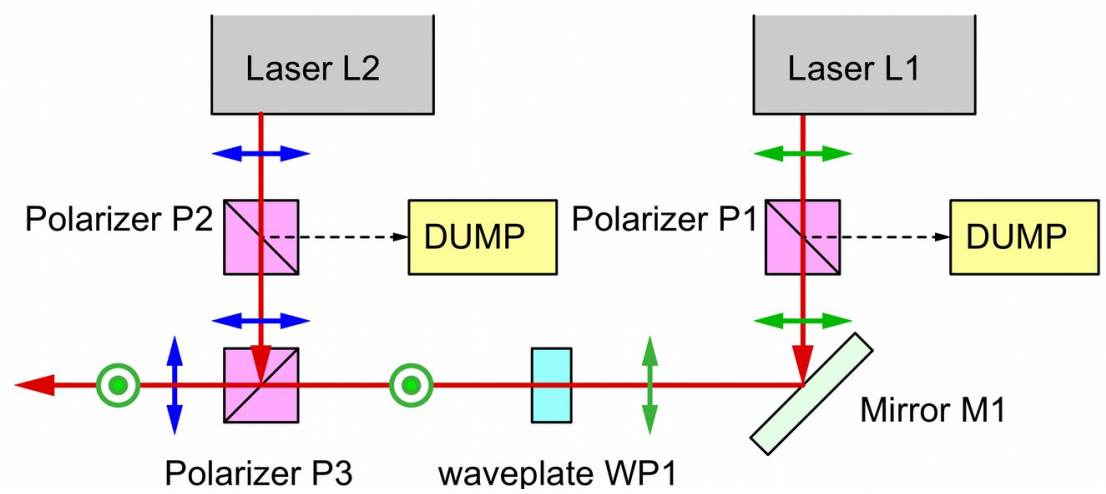

Fig. 2. Schematic diagram of the coaxial beam merging system.

\section{Light collection optics}

We have now two view windows as shown in Fig.3. First, the main view window was installed in the initial phase, and there is no significant change up to now. The main window is made from fused silica and the dimensions are 600 $\mathrm{mm}$ x $350 \mathrm{~mm}$ x $40 \mathrm{~mm}$. The distance between the LHD plasma center and the center of the main window, solid angle and scattering angle in the backscattering measurements are $3387 \mathrm{~mm}, 18 \mathrm{msr}$ and $167^{\circ}$ respectively. The Thomson scattered light is collected by a large, $1.8 \mathrm{~m} \times 1.5 \mathrm{~m}$, gold-coated mirror onto 144 optical fibers with the core diameter of $2.0 \mathrm{~mm}$. [7] The curvature of the spherical mirror is 4500 $\mathrm{mm}$, and the center of curvature has been

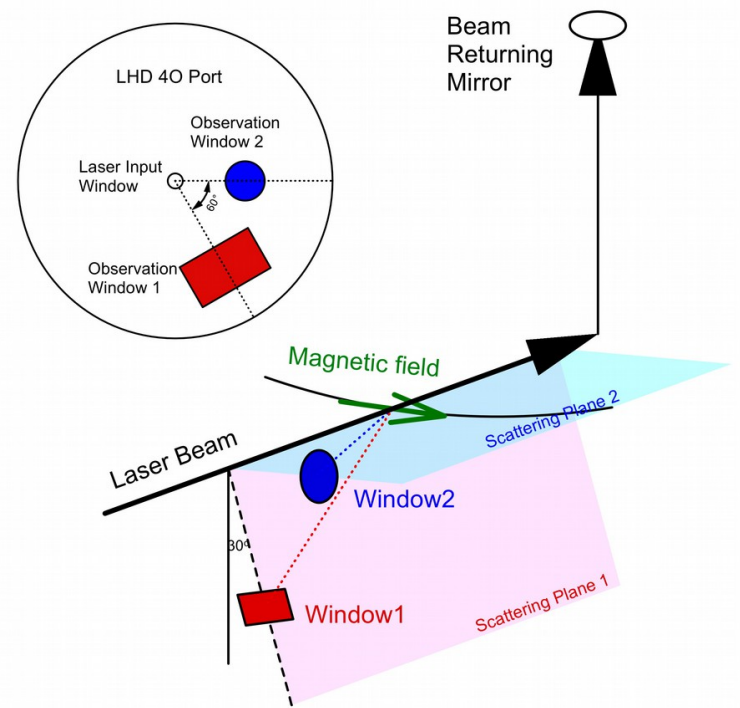

Fig.3. Schematic diagram of the two observation windows. 
set at the window center. In addition to the main window, we installed a small circular window, mirror and fiber to observe different electron temperature component as described later. The second view window is a circular fused silica window. The diameter, solid angle and scattering angle for the backward scattering measurement are $344 \mathrm{~mm}, 7.6 \mathrm{msr}$ and $171^{\circ}$ respectively. Currently the second light collection optics observes $T_{e}$ and $n_{e}$ at a spatial point (plasma center). By using the new window, $T_{e}$ component parallel to the LHD magnetic field line can be obtained around plasma center. The number of measurement points will be increased by adding optical fibers and polychromators.

Table I. Specification of the light collection optics.

\begin{tabular}{lcc} 
& Main window & Second window \\
Dimension [mm] & $350 \times 600$ & $344 \phi$ \\
Scattering angle [deg.] & $167^{\circ}$ & $171^{\circ}$ \\
Solid angle [msr] & 18 & 7.6 \\
Spatial resolution [mm] & 18 & 10 \\
Number of spatial points & 144 & 1 \\
\hline
\end{tabular}

\section{Polychromator}

We have operated three types of polychromators: low-temperature type (edge region), standard type (core region), and high-temperature type (central region). A schematic diagram of the polychromator is shown in Fig.4. [3] Thomson scattered photons are separated by the interference filters and detected by avalanche photo diode (APD) detectors. The wavelength channels, CH.1-5 are used for Thomson scattering measurement, and the final CH.6 is used for Rayleigh scattering calibration. Figures $5 \mathrm{a}$ ) and b) show examples of the spectral responses of a low-temperature type and standard type polychromators respectively. The solid curves show Thomson scattering spectra at $[5,50$ and $500 \mathrm{eV}]$ and $[100,1000$ and $5000 \mathrm{eV}]$ estimated by using the Selden's formula. [8] All polychromators are equipped with a Rayleigh scattering channel for absolute density calibration. [9] The standard type polychromators have been optimized for the temperature region of $50 \mathrm{eV} \leq T_{e} \leq 10 \mathrm{keV}$. The low-temperature type is designed for observing the low temperature edge region by changing the interference filters, and it can measure $T_{e}$ below $1 \mathrm{eV}$ to $500 \mathrm{eV}$. The peak transmittance of each filter is about $60-80 \%$, and the blocking power at $1064 \mathrm{~nm}$ is better than $10^{-4}$. In our Thomson scattering system, stray light is hardly detected, owing to the high blocking power In addition, the influence of plasma light is negligibly small in typical plasma experiments because high-pass filters (small capacitor) have been inserted between the preamplifier in the polychromator and the external DC amplifier. The cutoff frequency is $300 \mathrm{kHz}$. Therefore, a fast Thomson scattering signal with the pulse duration of $\sim 10 \mathrm{nsec}(\sim 100 \mathrm{MHz})$ can freely pass through the high-pass filter, and the slow plasma light component is almost completely rejected by the high-pass filter. However, the influence of intense plasma light on APDs sometimes appears in high-density experiments, resulting in strange results. We monitor the slow plasma light component too in order to check the data reliability. [10] It is noted that since the characteristics of band-pass filters and APDs strongly depend on the temperature, the temperature of the polychromators is stabilized with 
temperature-controlled $\left( \pm 0.1{ }^{\circ} \mathrm{C}\right)$ chilled water and the laboratory temperature is controlled within $1{ }^{\circ} \mathrm{C}$.

In order to obtain absolute electron densities, we have calibrated the total sensitivity of the light collection optics and polychromator by using Rayleigh scattering in gaseous nitrogen. We formerly tried both calibrations using Raman and Rayleigh scatterings. [9] In our case, Rayleigh scattering calibration provides more accurate and reliable calibration data, because the spectral response of our polychromators is not good for the accurate measurement of Raman scattering signals.

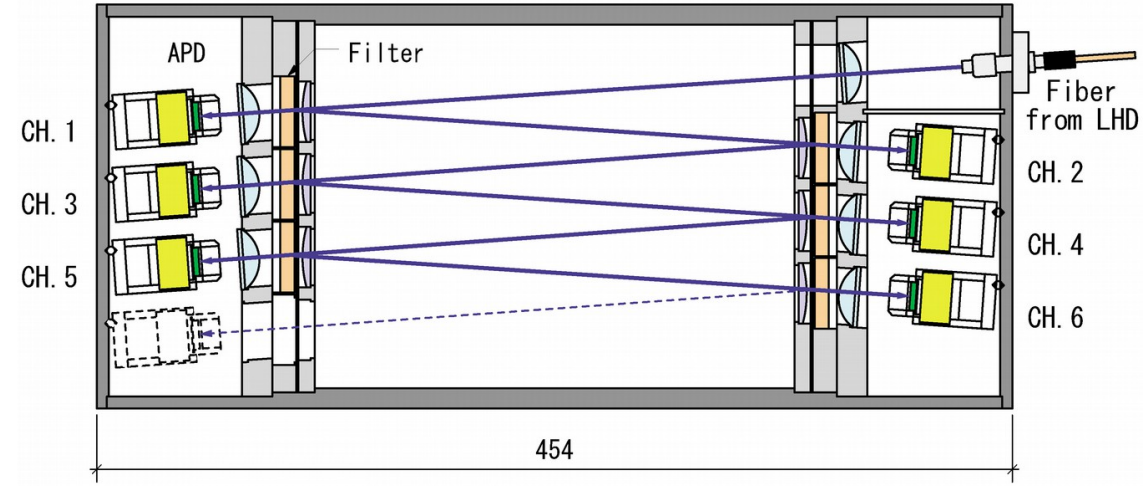

Fig. 4. Schematic diagram of the LHD polychromator.
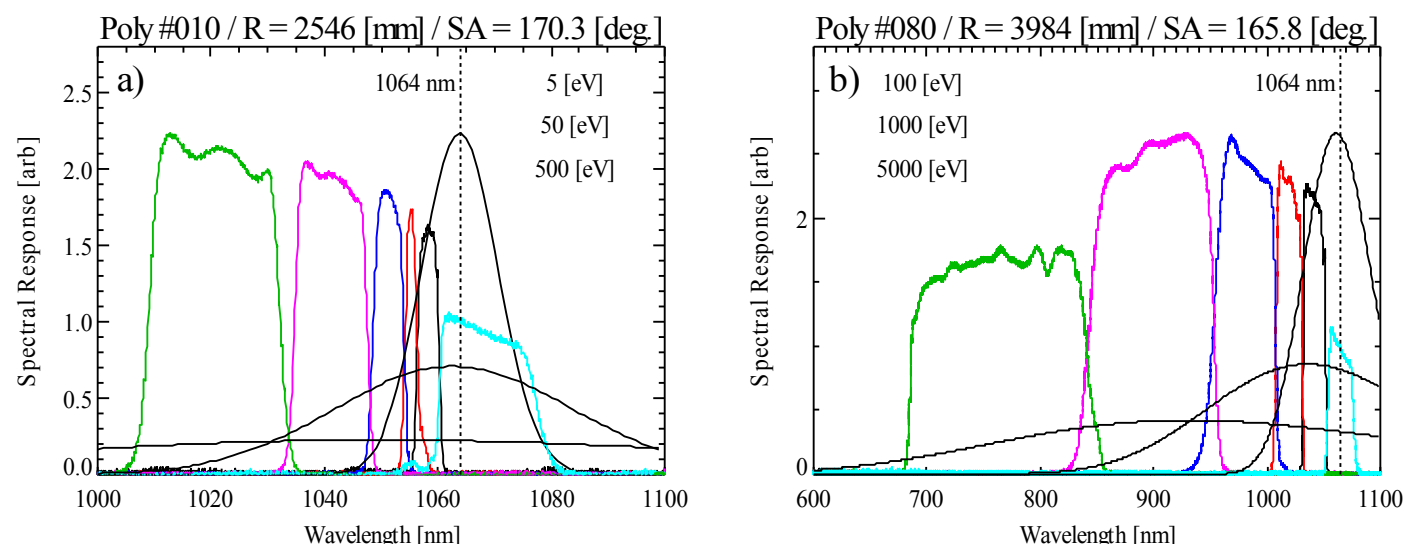

Fig. 5. Spectral response of the polychromators of the low- $T_{e}$ region, a), and core region, b), respectively.

\section{Scattering configuration}

The original LHD Thomson scattering system has a backward scattering configuration using the main view window. In the original design, laser pulses are absorbed by a beam dump after traveling through plasma. Recently we removed the beam dump and installed a beamreturning mirror and an optical delay path of $30 \mathrm{~m}$. [11] The laser beam is reflected by a mirror and runs through the LHD plasma again as shown in Fig. 6. Forward scattering light is emitted from the returned pulse toward the viewing window. The scattering angle is $13^{\circ}\left(=180^{\circ}-17^{\circ}\right)$ at the plasma center. The two Thomson scattering signals, backward and forward scatterings, can be separately observed due to the optical delay path. The temporal difference between the backward and forward scattering signals is $100 \mathrm{nsec}$. By combing the backward and forward scattering measurements, the measurable 
temperature range will be expanded as $T_{e}=$ $50 \mathrm{eV}-10 \mathrm{keV}$ to $T_{e}=1 \mathrm{eV}-30 \mathrm{keV}$. In addition, we have installed a new window as described in Section 2. We now can observe four $T_{e}$ components by using the two view windows and two scattering configurations, backward and forward Thomson scatterings. Detail analysis by comparing the four temperatures is now in progress. [12][13]

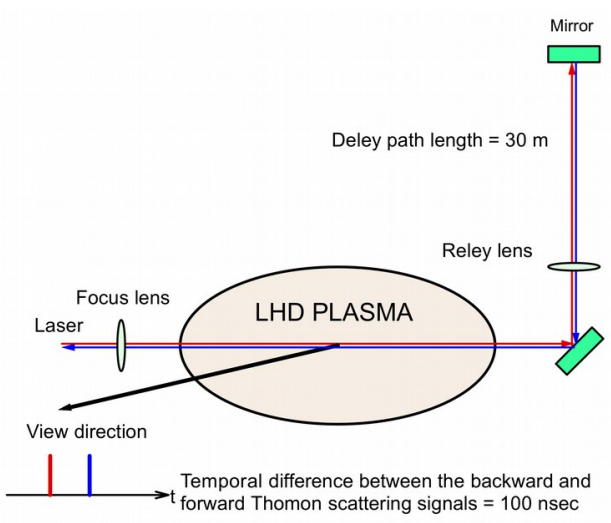

Fig. 6. Schematic diagram of the beam returning system.

\section{Summary}

The LHD Thomson scattering system has routinely measured electron temperature and density profiles of LHD plasmas since 1999. During the past 15 years, we have improved the LHD Thomson scattering system for increasing data reliability and accuracy, expanding measurable temperature and density ranges, and searching new physical findings. From the Thomson scattering measuremnets using the recently installed second view window and beam returning system, new insights of plasma physics will be provided.

This work was supported by the NIFS LHD project budgets (NIFS14ULHH005 and NIFS14ULHH801) and JSPS KAKENHI Grant Number 25289341.

\section{References}

[1] K. Narihara et al., Rev. Sci. Instrum., 72, 1122 (2001).

[2] K. Narihara et al., Phys. Rev. Lett., 87, 135002 (2002).

[3] Yamada et al., Fusion Sci. Tech., 58, 345 (2010).

[4] I. Yamada et al., Rev. Sci. Instrum., 72, 1126 (2001).

[5] T. N. Carlstorm et al.., Rev. Sci. Instrum., 63, 4901 (1992).

[6] R. Yasuhara et al., Plasma Fusion Res., 7, 2402030 (2012).

[7] K. Narihara et al., Rev. Sci. Instrum., 72, 3878 (2004).

[8] A. C. Selden, Phys. Lett., 79A, 405 (1980).

[9] I. Yamada et al., Plasma Fusion Res., S1106 (2007).

[10] K. Narihara et al., Plasma Fusion Res., 2, S1107 (2007).

[11] R. Yasuhra et al., Rev. Sci. Instrum. 83, 10E326 (2012).

[12] I. Yamada et al., 40 ${ }^{\text {th }}$ EPS Conf. Proc. O2.112 (2013).

[13] I. Yamada et al., 41 ${ }^{\text {th }}$ EPS Conf. Proc. P4.009 (2014). 